EPJ manuscript No.

(will be inserted by the editor)

\title{
Deuteron form factors in chiral effective theory: regulator- independent results and the role of two-pion exchange
}

\author{
Manuel Pavón Valderrama ${ }^{1}$ a, Andreas Nogga $^{1}$ b, Enrique Ruiz Arriola ${ }^{2}$, and Daniel R. Phillips ${ }^{3}$ d \\ 1 Institut für Kernphysik, Forschungszentrum Jülich, 52425 Jülich, Germany \\ 2 Departmento de Física Atómica, Molecular y Nuclear, Universidad de Granada, E-18071 Granada, Spain \\ 3 Department of Physics and Astronomy, Ohio University, Athens, OH 45701 \\ Received: date / Revised version: date
}

\begin{abstract}
We evaluate the deuteron charge, quadrupole, and magnetic form factors using wave functions obtained from chiral effective theory $(\chi \mathrm{ET})$ when the potential includes one-pion exchange, chiral two-pion exchange, and genuine contact interactions. We study the manner in which the results for form factors behave as the regulator is removed from the $\chi \mathrm{ET}$ calculation, and compare co-ordinate- and momentumspace approaches. We show that, for both the LO and NNLO chiral potential, results obtained by imposing boundary conditions in co-ordinate space at $r=0$ are equivalent to the $\Lambda \rightarrow \infty$ limit of momentum-space calculations. The regulator-independent predictions for deuteron form factors that result from taking the $\Lambda \rightarrow \infty$ limit using the LO $\chi \mathrm{ET}$ potential are in reasonable agreement with data up to momentum transfers of order $600 \mathrm{MeV}$, provided that phenomenological information for nucleon structure is employed. In this range the use of the NNLO $\chi \mathrm{ET}$ potential results in only small changes to the LO predictions, and it improves the description of the zero of the charge form factor.
\end{abstract}

PACS. 12.39.Fe Chiral Lagrangians - 25.30.Bf Elastic electron scattering - 21.45.+v Few-body systems

\section{Introduction}

Elastic electron scattering from the deuterium nucleus, the deuteron, has long been used as a means to assess the reliability of different nuclear forces. For recent reviews of experimental and theoretical work on the elastic electrondeuteron reaction see Refs. 1, 2,3. In this reaction the ability to vary the momentum transfer, $q$, to the nucleus means the electromagnetic structure of the deuteron can be probed on a variety of different scales. Indeed, in the "impulse approximation" - where two-nucleon currents are neglected - the matter distribution inside the nucleus is imaged, thereby providing a direct test of the $N N$ interaction used to predict it. In this paper we examine elastic electron-deuteron scattering for $q<1 \mathrm{GeV}$, where we anticipate that the deuteron's electromagnetic structure will be governed by the spontaneously and explicitly broken chiral symmetry of QCD.

This feature of QCD plays a role even though quark degrees of freedom are not resolved at this momentum scale. Indeed, confinement guarantees that for $q<1 \mathrm{GeV}$ it is useful to employ an effective field theory (EFT) in which nucleons and pions (and possibly baryon resonances) are the explicit degrees of freedom. Here we will use chiral perturbation theory $(\chi \mathrm{PT})$ which is an EFT in which the nucleons and pions interact with each other in a manner consistent with the chiral symmetry of QCD and the pattern of its breaking. $\chi \mathrm{PT}$ does not aim to describe hadronic and nuclear structure down to arbitrarily short distances, but is based on the inability of low-momentum-transfer probes to resolve the details of short-range interactions. It is thus ideally suited to the treatment of deuteron electromagnetic structure in the regime $q<1 \mathrm{GeV}$.

Chiral perturbation theory organizes the Lagrangian that describes nucleon and pion (and photon) interactions in powers of the small expansion parameter $P \equiv \frac{m_{\pi}, p}{\Lambda_{\chi \mathrm{SB}}}$, where $\Lambda_{\chi \mathrm{SB}} \sim m_{\rho}, 4 \pi f_{\pi}$ represents the scale of chiral-symmetry breaking, which is indicated by the $\rho$ mass and the pion decay constant, while $p$ is the momentum of the particles

a Email: m.pavon.valderrama@fz-juelich.de

b Email: a.nogga@fz-juelich.de

c Email: earriola@ugr.es

d Email: phillips@phy.ohiou.edu 
involved, and $m_{\pi}$ the pion mass. This theory has had considerable success in describing pion-nucleon and mesonmeson interactions [4,5. Weinberg proposed to extend $\chi \mathrm{PT}$ to few-nucleon systems by making a $\chi \mathrm{PT}$ expansion for the nucleon-nucleon interaction, $V$, and then taking the non-perturbative character of nuclear systems into account by solving the Schrödinger equation based on this potential 6, 7, 8. The result is quantum mechanics with fixed particle number for few-nucleon systems in which the potential (and other operators) has a $\chi \mathrm{PT}$ expansion. This has been dubbed chiral effective theory $(\chi \mathrm{ET})$. This approach is reviewed in Refs. $9,10,11,12$.

Recently there has been considerable controversy about the correct way to implement this approach [13, 14, 15 , 16. It has been argued that Weinberg's original power counting needs to be modified for the short-range or contact interaction piece of $V$ if one wishes to obtain regulator-independent predictions over a wide range of cutoffs, $\Lambda$. However, as shown already in Ref. [17, in the ${ }^{3} \mathrm{~S}_{1}-{ }^{3} \mathrm{D}_{1}$ channel the leading-order chiral potential can be renormalized using the single contact interaction it contains. The limit $\Lambda \rightarrow \infty$ can be taken, as has since been confirmed by several authors [14, 18, 16, 19. Thus the discussions of Ref. 13, 14, 15, 16, are not relevant to our results for leading-order (LO) $\chi \mathrm{ET}$, and we will not contribute to that discussion here.

However, the situation is not as clear once sub-leading corrections are included in the $N N$ interaction $V$. In particular, while one naively expects that these corrections to $V$ can be treated in perturbation theory, previous works have included them non-perturbatively, by solving the Schrödinger equation with the full $V$ at a fixed order in $\chi \mathrm{PT}$. In such a calculation the sub-leading contributions to $V$ dominate over the leading-order part of the $\chi \mathrm{PT}$ potential for $r \ll 1 / m_{\pi}$, and the singularity of $V$ at short distances therefore becomes more severe as the chiral order to which $V$ is computed is increased. In what follows we adopt such a non-perturbative treatment of $V$, even though it leads to a paradox when the next-to-leading-order $\left[O\left(P^{2}\right)\right] V$ is employed. In standard $\chi \mathrm{PT}$, where only nucleons and pions are taken as the relevant degrees of freedom, the next-to-leading order (NLO) $V$ calculated for $r \sim 1 / m_{\pi}$ is repulsive for $r \ll 1 / m_{\pi}$. As is well known from the theory of singular potentials [20, this means that the spectrum of the potential is predicted in the limit that the cutoff on the potential is removed, i.e. that the $\chi \mathrm{PT}$ potential is taken to be valid for all $r$. In consequence in the limit $\Lambda \rightarrow \infty$ there is no opportunity to tune the contact interaction and thereby reproduce the binding energy of the shallow $N N$ bound state in the ${ }^{3} \mathrm{~S}_{1}-{ }^{3} \mathrm{D}_{1}$ channel 21 .

Therefore, we can only present results for the LO $\left[O\left(P^{0}\right)\right]$ and next-to-next-to-leading order $\left[\mathrm{NNLO}=O\left(P^{3}\right)\right]$ chiral potentials $V$. Both of those potentials are attractive for $r \ll 1 / m_{\pi}$, which means that the inclusion of contact interaction(s) is mandatory if cutoff-independent predictions are to be obtained. Moreover, at these orders, and in the ${ }^{3} \mathrm{~S}_{1}-{ }^{3} \mathrm{D}_{1}$ channel of $N N$ scattering, there is no problem with Weinberg's original proposal: the three short-distance $N N$ operators his $\chi \mathrm{PT}$ expansion of $V$ predicts should be present at NNLO are sufficient to renormalize the attractive long-range potential generated by pion-nucleon dynamics at $r \sim 1 / m_{\pi}$.

In Section 2 we compute the deuteron wave functions obtained from the LO and NNLO chiral potentials $V$. We show that when we compute using a momentum-space cutoff on $V$ but demand that the deuteron binding energy be reproduced we obtain cutoff-independent predictions for the radial wave functions $u(r)$ and $w(r)$ for $r \gg 1 / \Lambda$. In the limit $\Lambda \rightarrow \infty$ the result of such a momentum-space calculation then agrees with that obtained via the co-ordinatespace approach advocated in Refs. [18,21]. We use existing wave functions to make this demonstration for the LO $\chi \mathrm{PT}$ potential $V$, and we also obtain, for the first time, NNLO momentum-space wave functions in $\chi \mathrm{ET}$ for $\Lambda>1$ $\mathrm{GeV}$. This allows us to make the connection between momentum- and co-ordinate-space not just at LO, but also at NNLO, and, by extension, for any order at which $V$ is attractive at short distances.

These wave functions, while not directly observable, do enter in the matrix elements of nuclear current operators that are probed in electron scattering from the deuterium nucleus. In particular, to the extent that the impulse approximation is valid, the form factors that determine all elastic electron-deuteron scattering observables in the onephoton-exchange approximation can be expressed as Bessel transforms of bilinears of these wave functions. We present results for these form factors in Sec. 3. These results are based on a chiral expansion of the deuteron current operators 22, 23. Up to relative order $P^{3}$ there are no two-nucleon contributions in either the charge operator, $J^{0}$ or the vectorpart of the current, $\mathbf{J}$, and the impulse approximation holds. The deuteron form factors have already been calculated in such an approach (including two-body currents) in Refs. 24, 25, 26, 27]. Here, we follow [26] and focus on tests of deuteron structure by computing the ratio $G_{C} / G_{E}^{(s)}$, where the denominator is the nucleon isoscalar form factor. We show that cutoff artifacts disappear in this ratio as $\Lambda \rightarrow \infty$, allowing us to obtain the first regulator-independent, $\mathrm{LO}$, results for $G_{C}$ in the literature. We find that if we use phenomenological input for $G_{E}^{(s)}$ these results are in good agreement with data for $G_{C}$.

If $\chi \mathrm{ET}$ is to be a systematic expansion for $N N$ interactions we should be able to also obtain results at higher orders in the $P$ expansion. Therefore in Sec. 4 we compute the results found for form factors when $G_{C}, G_{Q}$, and $G_{M}$ are computed with deuteron wave functions found using the NNLO $\chi \mathrm{PT}$ potential. This potential includes various contributions from two-pion-exchange mechanisms. Once again we find that the cutoff artifacts in observables vanish as $\Lambda \rightarrow \infty$. Importantly, we find that the shift from LO to NNLO results for the form factors is small for $q<600$ $\mathrm{MeV}$.

This suggests that if we could do a NLO calculation of deuteron structure it would be close to the regulatorindependent LO result in this kinematic domain. In the absence of a calculation at NLO with $\Lambda \rightarrow \infty$ in the theory 
with only explicit nucleon and pion degrees of freedom this is purely supposition. Nevertheless, we believe that our results with the NNLO $V$ and $\Lambda \rightarrow \infty$ give realistic estimates for two-pion-exchange contributions to the form factors. We hold this view because the $O\left(P^{3}\right)$ pieces of $V$ that make the difference between an NNLO and NLO calculation are numerically much more important than NLO pieces $28,29,30$. This is in large part because our NNLO $V$ includes the most important contributions of the $\Delta(1232)$ to the $N N$ interaction [28,31,30. In particular, if a consistent power counting for nuclear interactions requires taking the $\Delta(1232)$ into account as an explicit degree of freedom [28,31,32] our NNLO calculation will produce results similar to an NLO calculation in the theory with explicit Deltas.

The calculation presented in Sec. 4 shows significant sensitivity in the position of $G_{C}$ 's zero to the details of the NNLO $\chi \mathrm{PT}$ potential. However, any attempt to use data on the $q_{0}$ for which $G_{C}\left(q_{0}\right)=0$ to pin down the two-pionexchange part of the $N N$ force is clouded by the presence of two-nucleon pieces of $J_{0}$ at $O\left(P^{3}\right)$ - the same order relative to leading as that at which the two-pion-exchange pieces of $V$ first occur. Similar ambiguities bedevil attempts to use other features of deuteron electromagnetic form factors to identify aspects of the $N N$ force beyond one-pion

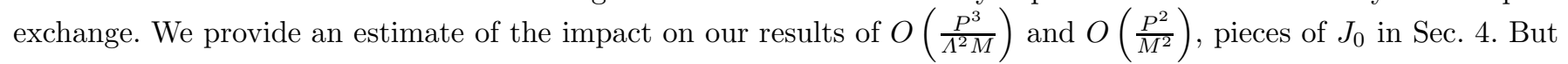
a full calculation of their contribution when $\Lambda \rightarrow \infty$ is beyond the scope of this paper. In Sec. [5, we summarize our findings and conclude.

\section{$2 \chi \mathrm{ET}$ wave functions: equivalence of co-ordinate- and momentum-space solutions}

$\chi \mathrm{ET}$ for few-nucleon systems results when $\chi \mathrm{PT}$ is applied to derive potentials and current operators which can then be used in a non-relativistic quantum-mechanical (fixed-particle-number) framework. Corrections to the static-nucleon picture may be treated in such a framework, e.g. here we incorporate the $1 / M$ corrections to $V$ as given by [29]. $1 / M^{2}$ pieces of $V$ and the effects of higher-Fock-space (e.g. $\pi N N$ ) states can also be incorporated, but will not concern us significantly here, although the former will be briefly discussed in Sec. 4 .

\subsection{Regulator-independent results for deuteron wave functions with the leading-order $\chi \mathrm{PT}$ potential}

As first discussed by Weinberg [6,7] the leading-order potential in the $\chi$ ET reproduces the time-honoured one-pion exchange (OPE) potential

$$
V^{(0)}\left(\boldsymbol{p}, \boldsymbol{p}^{\prime}\right)=-\frac{g_{A}^{2}}{4 f_{\pi}^{2}} \tau_{1} \cdot \tau_{2} \frac{\sigma_{1} \cdot\left(\mathbf{p}^{\prime}-\mathbf{p}\right) \sigma_{2} \cdot\left(\mathbf{p}^{\prime}-\mathbf{p}\right)}{\left(\mathbf{p}^{\prime}-\mathbf{p}\right)^{2}+m_{\pi}^{2}}+C_{S}+C_{T} \sigma_{1} \cdot \sigma_{2}
$$

We can now solve the Schrödinger equation in its momentum-space form, i.e. the homogeneous Lippmann-Schwinger equation:

$$
\left\langle\mathbf{p} \mid \psi_{\mathrm{LO}}\right\rangle_{\Lambda}=G_{0}(p) \int \frac{\mathrm{d}^{3} p^{\prime}}{(2 \pi)^{3}} V_{\Lambda}^{(0)}\left(\boldsymbol{p}, \boldsymbol{p}^{\prime}\right)\left\langle\mathbf{p}^{\prime} \mid \psi_{\mathrm{LO}}\right\rangle_{\Lambda},
$$

where $\Lambda$ is the scale at which the potential $V$ is regulated, and $G_{0}(p)=\left(-B_{d}-p^{2} / M\right)^{-1}$ is the (free, center-of-mass frame) two-nucleon propagator, with $B_{d}$ and $M$ denoting the deuteron binding energy and nucleon mass, respectively. The OPE contribution is determined through the pion mass $m_{\pi}=138 \mathrm{MeV}$, the axial coupling constant (for which we adopt $\left.g_{A}=1.29\right)$ and the pion-decay constant $f_{\pi}=92.4 \mathrm{MeV}$. In the triplet channel, only one linear combination of the contact interaction parameters, $C_{S}+C_{T}$, is relevant and is adjusted for each cutoff $\Lambda$ to reproduce the deuteron binding energy $B=2.225 \mathrm{MeV}$ as previously done, e.g., in 33. We perform the regularization using exponential cutoff functions

$$
V_{\Lambda}^{(0)}\left(\boldsymbol{p}, \boldsymbol{p}^{\prime}\right)=\exp \left(-\frac{p^{8}}{\Lambda^{8}}\right) V^{(0)}\left(\boldsymbol{p}, \boldsymbol{p}^{\prime}\right) \exp \left(-\frac{p^{\prime 8}}{\Lambda^{8}}\right) .
$$

Alternatively, the interaction can be Fourier-transformed to co-ordinate-space resulting in

$$
V(\mathbf{r})=\left[\tilde{C}_{S}+\tilde{C}_{T} \sigma_{1} \cdot \sigma_{2}\right] \delta^{(3)}(\mathbf{r})+\frac{g_{A}^{2}}{2 f_{\pi}^{2}} \sigma_{1} \cdot \nabla \sigma_{2} \cdot \nabla \frac{e^{-m_{\pi} r}}{r} .
$$

The Fourier transformation uniquely determines the long-range part of the interaction. (Note, however, that it involves a redefinition of the contact interactions to absorb a piece of $V^{(0)}$ that is a constant in momentum space.) The deuteron wave function in the pn CM system can be represented as

$$
\Psi(\boldsymbol{r})=\frac{1}{\sqrt{4 \pi} r}\left[u(r) \sigma_{p} \cdot \sigma_{n}+\frac{w(r)}{\sqrt{8}}\left(3 \sigma_{p} \cdot \hat{r} \sigma_{n} \cdot \hat{r}-\sigma_{p} \cdot \sigma_{n}\right)\right] \chi_{p n}^{s m_{s}}
$$


with the total spin $s=1$ and $m_{s}=0, \pm 1$ and $\sigma_{p}$ and $\sigma_{n}$ the Pauli matrices for the proton and the neutron, respectively. The functions $u(r)$ and $w(r)$ are the radial ${ }^{3} \mathrm{~S}_{1}$ and ${ }^{3} \mathrm{D}_{1}$ components of the relative wave function, respectively.

Even if we consider only $r>0$ and ignore the presence of the three-dimensional delta functions, this is a singular quantum-mechanical potential. In the absence of any short-distance regulator, the resulting Hamiltonian is unbounded from below and the spectrum contains an infinite number of bound states. This happens for any value of the parameters. On the other hand, it is known that physically there is only one bound $N N$ state: the deuteron. The presence of these unphysical, spurious bound states should not have any impact on low-energy observables, including the form factors at low momentum transfers. We will come back to this issue in Section 3 ,

To derive the wave function from Eq. (4), we use the representation of Eq. (5) and find the usual coupled onedimensional differential equations for the radial wave functions $u$ and $w$ in the presence of a tensor potential:

$$
\begin{aligned}
-u^{\prime \prime}(r)+U_{s}(r) u(r)+U_{s d}(r) w(r) & =-\gamma^{2} u(r), \\
-w^{\prime \prime}(r)+U_{s d}(r) u(r)+\left[U_{d}(r)+\frac{6}{r^{2}}\right] w(r) & =-\gamma^{2} w(r),
\end{aligned}
$$

The coupled-channel reduced potential $\left(U=2 \mu_{p n} V\right.$ with $\left.2 \mu_{p n}=2 M_{p} M_{n} /\left(M_{p}+M_{n}\right)\right)$ is given by

$$
U_{s}=U_{C}, \quad U_{s d}=2 \sqrt{2} U_{T}, \quad U_{d}=U_{C}-2 U_{T},
$$

with

$$
\begin{aligned}
& U_{C}=-\frac{m_{\pi}^{2} M g_{A}^{2}}{16 \pi f_{\pi}^{2}} \frac{e^{-m_{\pi} r}}{r}, \\
& U_{T}=-\frac{m_{\pi}^{2} M g_{A}^{2}}{16 \pi f_{\pi}^{2}} \frac{e^{-m_{\pi} r}}{r}\left(1+\frac{3}{m_{\pi} r}+\frac{3}{\left(m_{\pi} r\right)^{2}}\right),
\end{aligned}
$$

provided that $r>0$.

For our co-ordinate-space calculations, the equations (6) are solved subject to the boundary conditions

$$
\begin{aligned}
u(r) & \rightarrow A_{S} e^{-\gamma r}, \\
w(r) & \rightarrow \eta A_{S} e^{-\gamma r}\left(1+\frac{3}{\gamma r}+\frac{3}{(\gamma r)^{2}}\right),
\end{aligned}
$$

for $r \rightarrow \infty$. Here, $\gamma=\sqrt{M B_{d}}$ is the deuteron wave number, $A_{S}$ is a normalization constant and can be chosen to guarantee that

$$
\int_{0}^{\infty} \mathrm{d} r\left(u^{2}(r)+w^{2}(r)\right)=1
$$

and $\eta$ is the asymptotic $\mathrm{D} / \mathrm{S}$ ratio.

The form of the singularity of the OPE potential at short-distances implies that, for sufficiently small $r$, the components $u$ and $w$ are given by $34,18,35$ :

$$
\begin{aligned}
& u_{\text {short }}(r)=A_{S} \frac{1}{\sqrt{3}}\left(\frac{r}{R}\right)^{3 / 4}\left[-C_{2 R} e^{-4 \sqrt{2} \sqrt{R / r}}+2^{3 / 2}\left|C_{2 A}\right| \cos \left(4 \sqrt{\frac{R}{r}}+\phi\right)\right], \\
& w_{\text {short }}(r)=A_{S} \frac{1}{\sqrt{3}}\left(\frac{r}{R}\right)^{3 / 4}\left[\sqrt{2} C_{2 R} e^{-4 \sqrt{2} \sqrt{R / r}}+2\left|C_{2 A}\right| \cos \left(4 \sqrt{\frac{R}{r}}+\phi\right)\right] .
\end{aligned}
$$

$C_{2 A}$ and $C_{2 R}$ are normalization constants which have been determined in [18, $R$ is a new length scale that enters the non-perturbative problem, given by $R=\frac{3 g_{A}^{2} M}{32 \pi f_{\pi}^{2}}$. When Eqs. (6) -(8) are solved in Ref. [18] the phase $\phi$ is determined by the boundary condition at $r=0$, and so $\phi$ is regulator independent, and is a function only of the scales $m_{\pi}, \gamma$, and $R$.

The wave functions $u$ and $w$ can now be calculated by applying standard techniques to Eqs. (6) -(8) and employing the long-distance boundary conditions (9) 18. The asymptotic D-to-S ratio $\eta$ is then determined by the requirement that the numerical solution matches the short-distance behavior Eq. (11). In practice, this is done by imposing the additional boundary condition $u\left(r_{c}\right)-\sqrt{2} w\left(r_{c}\right)=0$, which is a direct consequence of Eq. (11), at a value of $r_{c} \ll R$. For this purpose we choose $r_{c}=0.1-0.2 \mathrm{fm}$ [18], and find $\eta_{\mathrm{OPE}}(\gamma) \approx 0.026333$.

The necessity to choose a finite $r_{c}$ leads to a small numerical uncertainty of the constants $C_{2 A}, C_{2 R}$ quoted in Ref. 18. These uncertainties can, however, be made arbitrarily small. Furthermore, any deuteron matrix element that 


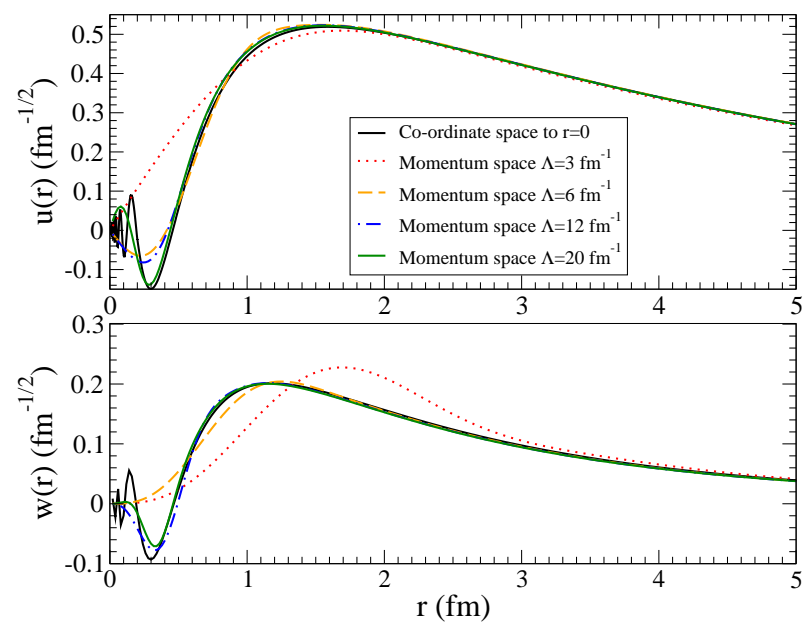

Figure 1. Comparison of radial deuteron wave functions $u(r)$ and $w(r)$ computed with the LO $\chi$ ET potential using a momentum-space regularization [14,33] and the co-ordinate-space regularization of Ref. [18].

is finite when computed with the wave functions $u$ and $w$ obtained in this way is insensitive to $r_{c}$ as long as $r_{c}$ is taken small enough [36]. Therefore, for all practical purposes, the wave functions found by the technique of Ref. [18] are the deuteron wave functions found with a strict contact interaction. The contact parameter $C_{S}+C_{T}$ has been converted to a boundary condition that is imposed at $r=0$ [37.

For non-singular interactions, it is clear that any solution obtained in momentum space will be the Fourier-transform of the corresponding co-ordinate-space solution. The question arises whether this holds for the solution of the singular OPE interaction based on Eq. (2). In momentum space the solution is only ever determined for a finite cutoff $\Lambda$, and it is not a priori clear that the limit $\Lambda \rightarrow \infty$ of the momentum-space wave function will, upon taking the Fourier transform, lead to the co-ordinate-space wave function found using Eqs. (6) -(8).

In Fig. I, we show that, for any $r>0$, the momentum-space solution does indeed approach this co-ordinate-space solution for $\Lambda \rightarrow \infty$. It is reassuring that one obtains equivalent results in both schemes. This confirms that fitting the contact interactions in a momentum-space calculation corresponds to imposing proper co-ordinate-space boundary conditions and matching to the short-distance form of the wave functions (11).

However, the relationship between $\Lambda$ and the numerically necessary co-ordinate-space cutoff $r_{c}$ remains somewhat opaque. For theories in which the long-range-part of the potential is absent one can establish that a sharp cut-off $\Lambda$ and the matching radius $r_{c}$ for the Schrödinger equation with a boundary condition are related by $r_{c}=\frac{\pi}{2 \Lambda}[38$. But here, given our choice of regulator and the presence of a long-range potential (one-pion exchange) this relation is no longer valid. Indeed, especially for $w(r)$, the wave function is only converged for distances

$$
r \gg \frac{\pi}{2 \Lambda} .
$$

Therefore we conclude that simple arguments for "reasonable" choices of the momentum-space cutoff $\Lambda$ at which results converge to the $\Lambda \rightarrow \infty$ limit can be misleading.

\subsection{Regulator-independent results for deuteron wave functions with the NNLO $\chi$ ET potential}

In Sec. 3 we use the (co-ordinate- and momentum-space) wave functions we have obtained thus far to predict the electromagnetic structure of the deuterium nucleus. However, this represents only a LO calculation in $\chi \mathrm{ET}$, so before performing such a calculation of deuterium electromagnetic form factors we will explain how the two-pion exchange (TPE) contribution to the $N N$ potential $V$ is incorporated in $\chi \mathrm{ET}$.

As outlined in the introduction, $\chi \mathrm{PT}$ can be used to expand the long-range $\left(r \sim 1 / m_{\pi}\right)$ part of the NN potential. Here we do this up to NNLO. The explicit expressions in momentum- and co-ordinate-space were given in Ref. [29. Note, however, that the momentum-space expressions of Ref. [29] are opposite in sign to ours, since different conventions are used. Also, it should be noted that we have used $g_{A}=1.26$ for the TPE part of the NNLO potential.

For the calculations in co-ordinate-space, we follow the formalism introduced by two of us in Ref. 21. where twopion-exchange effects were included non-perturbatively. For the NNLO interaction the short-range potential behaves 
Table 1. The four different sets of values for the chiral coefficients from $\mathcal{L}_{\pi N}^{(2)}$ that we consider in this work.

\begin{tabular}{c|c|c|c|c} 
Set & Source & $c_{1}\left(\mathrm{GeV}^{-1}\right)$ & $c_{3}\left(\mathrm{GeV}^{-1}\right)$ & $c_{4}\left(\mathrm{GeV}^{-1}\right)$ \\
\hline Set I & $\pi N[\underline{42}$ & -0.81 & -4.69 & 3.40 \\
Set II & $N N \underline{40}$ & -0.76 & -5.08 & 4.70 \\
Set III & $N N \underline{43}$ & -0.81 & -3.40 & 3.40 \\
Set IV & $N N \underline{44}$ & -0.81 & -3.20 & 5.40
\end{tabular}

like $29,39,40$

$$
\begin{aligned}
U_{s}^{\mathrm{TPE}}(r) & \rightarrow \frac{R_{s}^{4}}{r^{6}}, \\
U_{s d}^{\mathrm{TPE}}(r) & \rightarrow \frac{R_{s d}^{4}}{r^{6}}, \\
U_{d}^{\mathrm{TPE}}(r) & \rightarrow \frac{R_{d}^{4}}{r^{6}}
\end{aligned}
$$

where

$$
\begin{aligned}
\left(R_{s}\right)^{4} & =\frac{3 g_{A}^{2}}{128 f_{\pi}^{4} \pi^{2}}\left(4-3 g_{A}^{2}+24 \bar{c}_{3}-8 \bar{c}_{4}\right), \\
\left(R_{s d}\right)^{4} & =-\frac{3 \sqrt{2} g_{A}^{2}}{128 f_{\pi}^{4} \pi^{2}}\left(-4+3 g_{A}^{2}-16 \bar{c}_{4}\right) \\
\left(R_{d}\right)^{4} & =\frac{9 g_{A}^{2}}{32 f_{\pi}^{4} \pi^{2}}\left(-1+2 g_{A}^{2}+2 \bar{c}_{3}-2 \bar{c}_{4}\right)
\end{aligned}
$$

and $\bar{c}_{i}=M c_{i}$ are the low energy chiral couplings (LECs) appearing in $\pi N$ scattering 41. We will present results for the sets of $c_{i}$ parameters shown in Table 1, which are the same four sets as were used in Ref. [13. In contrast to OPE, this interaction yields two attractive eigenchannels upon diagonalization. Therefore, the short-distance wave functions in the ${ }^{3} \mathrm{~S}_{1}$ and ${ }^{3} \mathrm{D}_{1}$ channels take the form [21]:

$$
\begin{aligned}
& u_{\text {short }}(r)=\left\{C_{+}\left(\frac{r}{R_{+}}\right)^{\frac{3}{2}} \cos \theta \sin \left[\frac{1}{2} \frac{R_{+}^{2}}{r^{2}}+\phi_{+}\right]+C_{-}\left(\frac{r}{R_{-}}\right)^{\frac{3}{2}} \sin \theta \sin \left[\frac{1}{2} \frac{R_{-}^{2}}{r^{2}}+\phi_{-}\right]\right\} \\
& w_{\text {short }}(r)=\left\{-C_{+}\left(\frac{r}{R_{+}}\right)^{\frac{3}{2}} \sin \theta \sin \left[\frac{1}{2} \frac{R_{+}^{2}}{r^{2}}+\phi_{+}\right]+C_{-}\left(\frac{r}{R_{-}}\right)^{\frac{3}{2}} \cos \theta \sin \left[\frac{1}{2} \frac{R_{-}^{2}}{r^{2}}+\phi_{-}\right]\right\}
\end{aligned}
$$

where the coefficients $C_{+}$and $C_{-}$and the phases $\phi_{+}$and $\phi_{-}$are arbitrary. The scales $R_{+}$and $R_{-}$and the angle $\theta$ are determined by diagonalizing the potential Eq. (13). Because the short-distance wave functions (15) do not constrain the two components $u(r)$ and $w(r)$ as strongly as Eq. (11) does in the case of OPE, any solution based on the Schrödinger equation (6) and the long-distance boundary conditions Eq. (9) can be matched to the $r \ll R_{-}, R_{+}$wave function. Apart from an overall normalization, the long-distance wave function of the deuteron depends on two parameters, $\gamma$ and $\eta$. Once these parameters have been fixed, e.g. by experiment, we are able to calculate the wave function to arbitrarily small distances $r>0$ uniquely and regulator-independently.

The numerical solution can thus be used to determine the parameters of the short-distance solution (15). In this way $\gamma$ and $\eta$ are linked to two of the contact interactions that are present in the NNLO $\chi$ PT potential. The third contact interaction does not affect the bound state, but can be determined by an examination of continuum wave functions. For example, for Set IV of the LECs we have $R_{+}=2.11 \mathrm{fm}$ and $R_{-}=1.16 \mathrm{fm}$. Choosing the normalization of the long distance wave function such that $u(r) \rightarrow e^{-\gamma r}$, and fixing $\gamma$ to its experimental value, the dependence of $C_{+,-}$and $\phi_{+,-}$on $\eta$ can be computed. The expressions can be found in Ref. 21], where an explicit expression for the dependence of $A_{S}$ on $\eta$ for the case of this potential is also given.

Since the dominant singularity of the NNLO $\chi \mathrm{PT}$ potential is $r^{-6}$ the solution of the ${ }^{3} \mathrm{~S}_{1}-{ }^{3} \mathrm{D}_{1}$ bound-state problem when this potential is used for all $r$ leads to a deuteron wave function that approaches zero more rapidly as $r \rightarrow 0$ than does the LO $\chi \mathrm{ET}$ wave function. As a consequence, deuteron matrix elements computed with wave functions where the NNLO potential is treated non-perturbatively have improved ultraviolet convergence properties [48. In particular, matrix elements such as $\langle 1 / r\rangle$ and $\left\langle 1 / r^{2}\right\rangle$, which are somewhat sensitive to short-distance pieces of the 


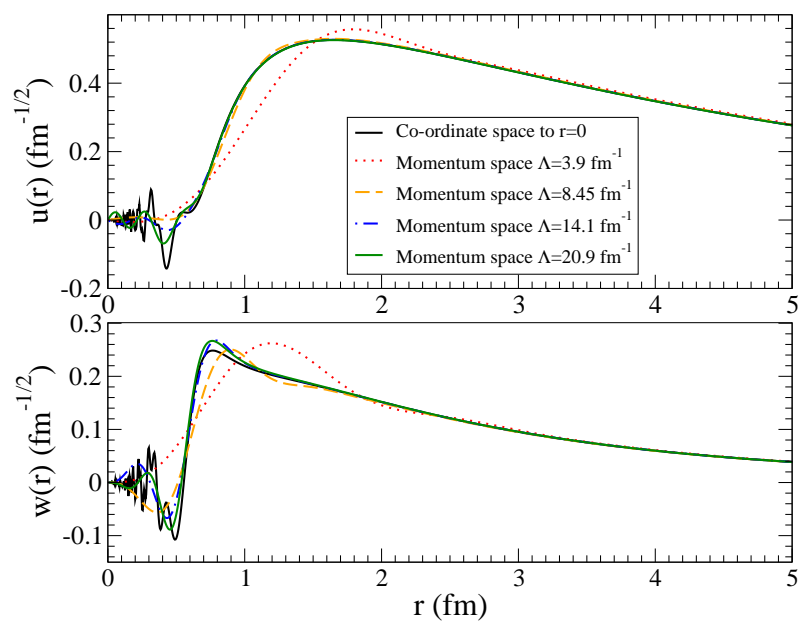

Figure 2. Comparison of radial deuteron wave functions $u(r)$ and $w(r)$ computed with the NNLO $\chi \mathrm{ET}$ potential using a momentum-space regularization and the co-ordinate-space regularization of Ref. [21].

wave function, take values that agree quite well those found when sophisticated $N N$ potential models are employed for their evaluation.

Now we turn our attention to momentum-space solutions of Eq. (2) with the NNLO TPE potential derived in Refs. 28, 29. We adjust the momentum-independent contact interaction $C_{S}+C_{T}$ so that the experimental deuteron binding energy is reproduced. A numerical Fourier transform allows us to determine $\eta$ as a function of the cutoff $\Lambda$. We find that for cutoffs $\Lambda \approx 3.9 \mathrm{fm}^{-1}, 8.45 \mathrm{fm}^{-1}, 14.1 \mathrm{fm}^{-1}$ and $20.9 \mathrm{fm}^{-1}$, the central value of the experimental $\mathrm{D} / \mathrm{S}$ ratio, $\eta=0.0256$ [45, is reproduced. Therefore at these values of the cutoff the coefficient of the second contact interaction needed to solve the ${ }^{3} \mathrm{~S}_{1}-{ }^{3} \mathrm{D}_{1}$ bound-state problem can be chosen to be zero.

When the momentum-space problem is solved in this way and the momentum-space wave functions are (numerically) transformed to co-ordinate-space the long-distance behavior matches that obtained in Ref. [21. Therefore, for these cutoffs, we should find equivalent solutions in momentum and co-ordinate-space in the region where $r \gg \frac{\pi}{2 \Lambda}$.

In Fig. 2, we compare the Fourier-transformed wave functions of the momentum-space calculations and the regulator-independent result of the co-ordinate-space calculation. For the cutoff range that we consider here, we find agreement down to radii $r \approx 1 \mathrm{fm}$. We hypothesize that if $\Lambda \rightarrow \infty$ the wave functions agree for all $r>0$. But as before, the momentum-space cutoffs need to be surprisingly large to find good agreement. E.g., for cutoff $\Lambda \approx 3.9 \mathrm{fm}^{-1}$, there are visible deviations to the r-space wave function at $r \approx 3 \mathrm{fm}$, and even for the largest cutoffs we observe deviations in the peak structure of $w(r)$ around $r \approx 1 \mathrm{fm}$. In the next section we will test whether this deviation has any noticeable effect on deuteron electromagnetic form factors.

\section{Deuteron form factors at leading order in $\chi \mathrm{ET}$}

Elastic electron-deuteron scattering can be parameterized by three independent form factors [1,2,3], which we will here write as Breit-frame matrix elements of the two-nucleon four-current $J_{\mu}$ :

$$
\begin{aligned}
G_{C} & =\frac{1}{3|e|}\left(\left\langle 1\left|J^{0}\right| 1\right\rangle+\left\langle 0\left|J^{0}\right| 0\right\rangle+\left\langle-1\left|J^{0}\right|-1\right\rangle\right), \\
G_{M} & =-\frac{1}{\sqrt{2 \eta}|e|}\left\langle 1\left|J^{+}\right| 0\right\rangle, \\
G_{Q} & =\frac{1}{2|e| \eta M_{d}^{2}}\left(\left\langle 0\left|J^{0}\right| 0\right\rangle-\left\langle 1\left|J^{0}\right| 1\right\rangle\right) .
\end{aligned}
$$

The form factors defined in Eqs. (16)-(18) are related to the static moments of the nucleus by:

$$
\begin{aligned}
G_{C}(0) & =1 \\
G_{Q}(0) & =Q_{d} \\
G_{M}(0) & =\mu_{d} \frac{M_{d}}{M}
\end{aligned}
$$


with $M_{d}$ the deuteron mass, $Q_{d}$ the deuteron quadrupole moment, and $\mu_{d}$ the deuteron magnetic moment in units of nuclear magnetons. In Eqs. (16)-(18), we have labeled the deuteron states by the projection of the deuteron spin along the direction of the three-vector $\mathbf{p}_{e}^{\prime}-\mathbf{p}_{e} \equiv \mathbf{q}$, and $\eta \equiv Q^{2} /\left(4 M_{d}^{2}\right)$, with $Q^{2}=|\mathbf{q}|^{2}$ since we are in the Breit frame. We can then calculate the deuteron structure functions:

$$
\begin{aligned}
A & =G_{C}^{2}+\frac{2}{3} \eta G_{M}^{2}+\frac{8}{9} \eta^{2} M_{d}^{4} G_{Q}^{2}, \\
B & =\frac{4}{3} \eta(1+\eta) G_{M}^{2} .
\end{aligned}
$$

In terms of $A$ and $B$, the one-photon-exchange interaction yields a lab-frame differential cross section for unpolarized electron-deuteron scattering [2]

$$
\frac{d \sigma}{d \Omega}=\frac{\sigma_{\mathrm{Mott}}}{1+\frac{2 E}{M_{d}} \sin ^{2}\left(\frac{\theta_{e}}{2}\right)}\left[A\left(Q^{2}\right)+B\left(Q^{2}\right) \tan ^{2}\left(\frac{\theta_{e}}{2}\right)\right] .
$$

Here $\theta_{e}$ is the electron scattering angle and $E$ the electron energy, and $\sigma_{\text {Mott }}$ is the Mott cross section. Measurement of the differential cross section only yields information on $G_{M}$ and a combination of $G_{C}$ and $G_{Q}$. A third observable (usually $t_{20}$, a tensor polarization observable which is sensitive to $G_{Q} / G_{C}$ ) must be measured if all three form factors are to be disentangled, and electron-deuteron scattering realize its full potential as a tool for measuring the deuteron's four-current.

If we make a chiral expansion for the deuteron current operator [24,25,26] the results, up to corrections suppressed by three powers of the $\chi \mathrm{PT}$ expansion parameter $P \equiv \frac{p, m_{\pi}}{\Lambda}$, can be written as:

$$
\begin{aligned}
\left\langle\mathbf{p}^{\prime}\left|J_{0}(\mathbf{q})\right| \mathbf{p}\right\rangle & =|e| G_{E}^{(s)}\left(Q^{2}\right) \delta^{(3)}\left(\mathbf{p}^{\prime}-\mathbf{p}-\mathbf{q} / \mathbf{2}\right), \\
\left\langle\mathbf{p}^{\prime}|\mathbf{J}(\mathbf{q})| \mathbf{p}\right\rangle & =\left[|e| \frac{\mathbf{p}+\mathbf{q} / 4}{M} G_{E}^{(s)}\left(Q^{2}\right)+i \mu_{S} \sigma \times \mathbf{q} G_{M}^{(s)}\left(Q^{2}\right)\right] \delta^{(3)}\left(\mathbf{p}^{\prime}-\mathbf{p}-\mathbf{q} / \mathbf{2}\right),
\end{aligned}
$$

with $G_{E}^{(s)}$ and $G_{M}^{(s)}$ the isoscalar form factors of the nucleon, and $\mu_{S}$ the isoscalar nucleon magnetic moment. In obtaining these equations we have dropped corrections to $J_{\mu}$ that have coefficients $1 / M^{2}$. We will briefly discuss such "relativistic" corrections to the deuteron current operator in Sec. 4.

Sandwiching the operators (25) and (26) between deuteron states yields the following co-ordinate-space integrals for the deuteron form factors [1:

$$
\begin{aligned}
G_{C}\left(Q^{2}\right)= & G_{E}^{(s)}\left(Q^{2}\right) \int d \mathrm{r}\left[u^{2}(r)+w^{2}(r)\right] j_{0}\left(\frac{|\mathbf{q}| r}{2}\right) \\
G_{Q}\left(Q^{2}\right)= & G_{E}^{(s)}\left(Q^{2}\right) \frac{6 \sqrt{2}}{Q^{2}} \int d \mathrm{r}\left[u(r) w(r)-\frac{w^{2}(r)}{\sqrt{8}}\right] j_{2}\left(\frac{|\mathbf{q}| r}{2}\right) \\
\frac{2 M}{M_{d}} G_{M}\left(Q^{2}\right)= & G_{E}^{(s)}\left(Q^{2}\right) \frac{3}{2} \int d \mathrm{r} w^{2}(r)\left[j_{0}\left(\frac{|\mathbf{q}| r}{2}\right)+j_{2}\left(\frac{|\mathbf{q}| r}{2}\right)\right] \\
& +G_{M}^{(s)}\left(Q^{2}\right) 2 \int d \mathrm{r} u^{2}(r) j_{0}\left(\frac{|\mathbf{q}| r}{2}\right) \\
& +G_{M}^{(s)}\left(Q^{2}\right)\left\{\sqrt{2} \int d \mathrm{r} u(r) w(r) j_{2}\left(\frac{|\mathbf{q}| r}{2}\right)-\int d \mathrm{r} w^{2}(r)\left[j_{0}\left(\frac{|\mathbf{q}| r}{2}\right)-j_{2}\left(\frac{|\mathbf{q}| r}{2}\right)\right]\right\}
\end{aligned}
$$

where $j_{0}$ and $j_{2}$ are spherical Bessel functions.

We have demonstrated in Sec. 2 that we can determine regulator-independent deuteron wave functions in coordinate space. They may be used to compute the integrals Eqs. (27)-(29). At the same time, for any finite value of $\Lambda$, we can evaluate the form factors $G_{C}, G_{Q}$, and $G_{M}$ using the wave functions obtained by solution of Eqs. (11) and (2). In the following, we present the results as a function of $|\mathbf{q}|=\sqrt{Q^{2}}$ following the convention of Ref. [26]. We restrict ourselves to $|\mathbf{q}|$ below $1 \mathrm{GeV}$. This should cover essentially the whole range where $\chi \mathrm{PT}$ is expected to converge.

We do this within the context of a chiral expansion for ratios of deuteron and nucleon form factors, e.g. $G_{C} / G_{E}^{(s)}$, so that we can focus on the predictions of $\chi \mathrm{ET}$ for deuteron structure. Data for the single-nucleon form factors could then be employed to generate final results for deuteron form factors. But, up to the chiral order to which we work, this procedure is equivalent to using phenomenological input for $G_{E}^{(s)}$ and $G_{M}^{(s)}$ in the evaluation of expressions for $G_{C}, G_{M}$, and $G_{Q}$, e.g. those in Eqs. (27)-(29). Therefore we simply use the phenomenological single-nucleon form 


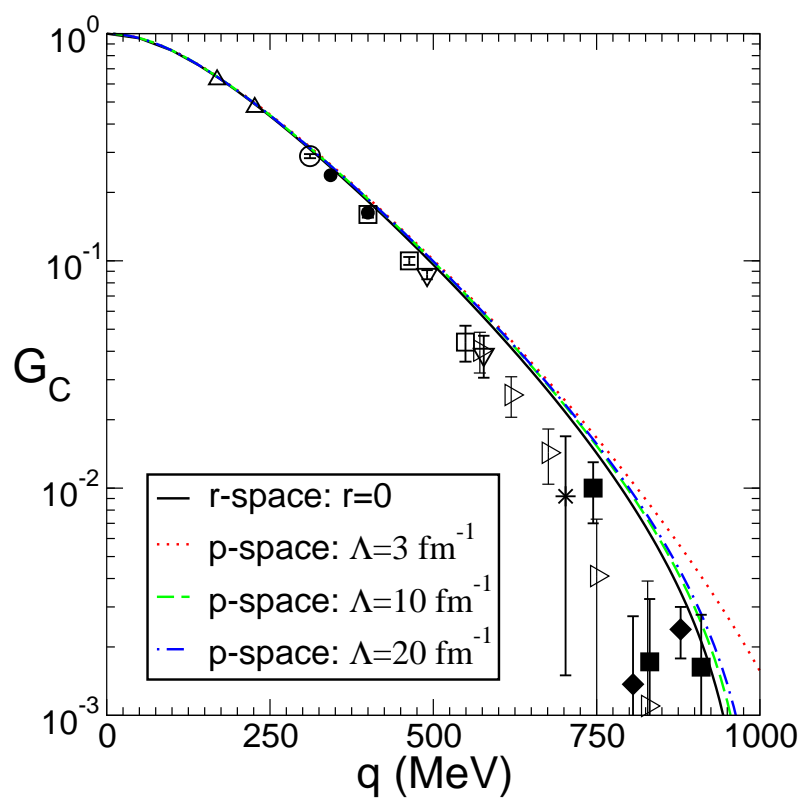

Figure 3. Charge form factor of deuterium, $G_{C}$, obtained using different regularizations of the leading-order $\chi \mathrm{ET}$ potential. The red dotted line, green dashed, and blue dot-dashed lines are for momentum-space calculations at $\Lambda=3,10$, and 20 $\mathrm{fm}^{-1}$, respectively. The black solid line is the co-ordinate-space result. The experimental data is taken from the compilation of Ref. [51]: upward triangles represent data from the $T_{20}$ measurement of Ref. [53], open circle [54, solid circle [55], open squares [56], downward triangles [57], rightward triangles [52], star [58], solid squares [59], solid diamonds 60].

factors of Ref. [49] in our evaluation of the deuteron form factors. These single-nucleon form factors are based on dispersion relations, and have the practical advantage of quantifying error bands due to uncertainties in the input experimental data. If we instead use a strict $\chi \mathrm{PT}$ expansion for the nucleon form factors our predictions for deuteron form factors begin to deviate from data for $|\mathbf{q}| \sim 300 \mathrm{MeV}$ [25, 26. However, this deviation has nothing to do with deuteron structure. Instead it is associated with the restricted range of $|\mathbf{q}|$ over which $\chi \mathrm{PT}$ provides a valid description of nucleon structure. This is the reason that we employ the single-nucleon form factors of Ref. [4] as an input in our calculation. Such a procedure is consistent as regards our $\chi$ ET examination of electron-deuteron scattering because deuteron and nucleon electromagnetic structure factorize in this reaction up to corrections that are $O\left(P^{4}\right)[26$.

We first look at $G_{C}$ based on the OPE wave functions: see Fig. 3. For $\Lambda \rightarrow \infty$, the co-ordinate- and momentumspace wave functions yield essentially the same result. The approach to the $\Lambda \rightarrow \infty$ limit is also shown in Fig. 3 and is quite interesting. At $\Lambda=10 \mathrm{fm}^{-1}$ the form factor has converged to the $\Lambda \rightarrow \infty$ result. However, the result for $\Lambda=3 \mathrm{fm}^{-1}$ predicts a significantly different position of the zero of $G_{C}$. As we shall see below, this feature is particularly affected by higher-order corrections. The counterterm that would remove this $\Lambda$ dependence is one such effect, although it is of very high order: $O\left(e P^{5}\right)$. Small contributions of higher order will have most pronounced effects at such a zero, where all lower-order effects cancel by definition, and so sensitivity to the regulator scale in the vicinity of the minimum is not surprising. We note that for $|\mathbf{q}|<600 \mathrm{MeV}$, the difference between $\Lambda=3 \mathrm{fm}^{-1}$ and $\Lambda=20 \mathrm{fm}^{-1}$ results is never more than a few per cent.

The results obtained in co-ordinate-space and using large cutoffs in momentum space also agree very well for $G_{Q}$. As seen in Fig. (4. convergence of the momentum-space results to the $\Lambda \rightarrow \infty$ limit is even more rapid there. Presumably, this is because, as was observed in Ref. [50], $G_{Q}$ is less sensitive to short-distance details than is $G_{C}$. There is, however, one exception to this: the value of $G_{Q}$ at $Q^{2}=0$, i.e. the deuteron quadrupole moment, has significant evolution with $\Lambda$, dropping by more than $10 \%$ between $\Lambda=3 \mathrm{fm}^{-1}$ and $\Lambda=\infty$. This can be seen in Table 2 , where, for completeness, we also compile some other basic properties of our deuteron wave functions. As was observed in Ref. [27, such $\Lambda$-dependence is symptomatic that the $O\left(e P^{5}\right)$ counterterm impacts $Q_{d}$ more significantly than one would naively expect. A counterterm of natural size at $O\left(e P^{5}\right)$ can absorb this large a cutoff dependence, and once it does, the remaining short-distance effects in $G_{Q}$ are minimal below $|\mathbf{q}|=600 \mathrm{MeV}[27$.

In $G_{M}$ the $\Lambda \rightarrow \infty$ limit again yields the co-ordinate-space result. But the results for low cutoffs deviate from the $\Lambda \rightarrow \infty$ limit more than in any of the other form factors, as displayed in Fig. [5. They differ by $10 \%$ at $|\mathbf{q}|=600 \mathrm{MeV}$, which corresponds to probing momenta of $|\mathbf{q}| / 2=300 \mathrm{MeV}$ in the nucleus itself. This sensitivity is entirely consistent with the presence of a counterterm for $G_{M}$ at $O\left(e P^{4}\right)$, which is only $\mathrm{N}^{2} \mathrm{LO}$. Indeed, our calculation of $G_{M}$ includes all NLO effects in the deuteron three-current $\mathbf{J}$ (apart from small $1 / M^{2}$ corrections) so the counterterm would appear in $\mathbf{J}$ at the next order beyond what is presented here. 


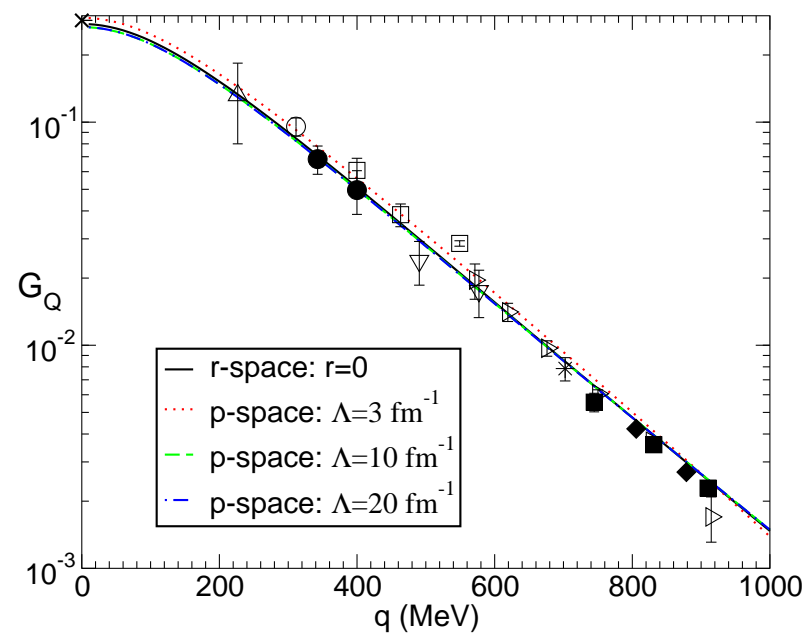

Figure 4. Quadrupole form factor of deuterium, $G_{Q}$, obtained using different regularizations of the leading-order $\chi \mathrm{ET}$ potential. Legend for theory curves as in Fig. 3] Experimental data taken from Ref. [51].

Table 2. Cutoff and scheme dependence of the kinetic energy $T$ in $\mathrm{MeV}$, the deuteron D-state probability $P_{D}$ in $\%$, the rms radius $r_{d}$ in $\mathrm{fm}$, the quadrupole moment $Q_{d}$ in $\mathrm{fm}^{2}$, the asymptotic S-state normalization in fm ${ }^{-1 / 2}$ and the asymptotic $\mathrm{S} / \mathrm{D}$ ratio $\eta$. The binding energy was fixed to $B=2.225 \mathrm{MeV}$ in all cases. For wave functions obtained in momentum space the cutoff $\Lambda$ is given in $\mathrm{fm}^{-1}$ while the r-space cut-off $r_{c}$ is in fm. The first two sets of results are based on OPE, while the lower rows are results for TPE (with Set IV $c_{i}^{\prime} s$ ). Experimental values for observable quantities are listed in the last row, together with references.

\begin{tabular}{|c|c|c|c|c|c|c|}
\hline$\Lambda$ & $T$ & $P_{D}$ & $r_{d}$ & $Q_{d}$ & $A_{S}$ & $\eta$ \\
\hline$\overline{2}$ & $\bar{~} 15.43$ & 6.98 & 1.90 & 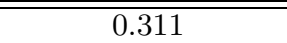 & $\overline{00.845}$ & 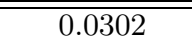 \\
\hline 3 & 19.49 & 8.82 & 1.94 & 0.299 & 0.869 & 0.0279 \\
\hline 5 & 28.41 & 7.03 & 1.95 & 0.279 & 0.873 & 0.0263 \\
\hline 10 & 39.62 & 7.29 & 1.94 & 0.277 & 0.869 & 0.0263 \\
\hline 14 & 46.95 & 7.27 & 1.94 & 0.277 & 0.869 & 0.0263 \\
\hline 20 & 57.40 & 7.27 & 1.93 & 0.276 & 0.868 & 0.0263 \\
\hline \multicolumn{7}{|c|}{$r$-space } \\
\hline 1.30 & 10.1 & 10.08 & 2.06 & 0.359 & 0.925 & 0.0302 \\
\hline 1.03 & 16.97 & 8.95 & 1.99 & 0.312 & 0.894 & 0.0279 \\
\hline 0.80 & 25.40 & 8.07 & 1.96 & 0.288 & 0.877 & 0.0268 \\
\hline 0.40 & 33.48 & 7.21 & 1.94 & 0.277 & 0.870 & 0.0263 \\
\hline 0.20 & 61.74 & 7.29 & 1.94 & 0.276 & 0.868 & 0.0263 \\
\hline 0.10 & 89.0 & 7.31 & 1.94 & 0.276 & 0.868 & 0.0263 \\
\hline \multicolumn{7}{|l|}{ 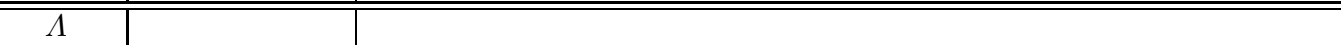 } \\
\hline 3.90 & 30.27 & 8.86 & 2.00 & 0.286 & 0.898 & 0.0257 \\
\hline 8.45 & 41.60 & 7.67 & 1.97 & 0.276 & 0.888 & 0.0254 \\
\hline 8.57 & 46.61 & 8.96 & 1.97 & 0.280 & 0.883 & 0.0260 \\
\hline 14.1 & 55.24 & 8.31 & 1.97 & 0.277 & 0.884 & 0.0256 \\
\hline 20.9 & 69.87 & 8.46 & 1.96 & 0.277 & 0.883 & 0.0256 \\
\hline \multicolumn{7}{|c|}{$r$-space } \\
\hline 0.92 & 22.93 & 6.72 & 2.00 & 0.286 & 0.898 & 0.0257 \\
\hline 0.67 & 39.45 & 7.36 & 1.98 & 0.276 & 0.888 & 0.0254 \\
\hline 0.66 & 46.74 & 8.85 & 1.97 & 0.281 & 0.883 & 0.0260 \\
\hline 0.50 & 59.53 & 8.06 & 1.97 & 0.277 & 0.885 & 0.0256 \\
\hline 0.35 & 112.8 & 8.13 & 1.97 & 0.277 & 0.884 & 0.0256 \\
\hline 0.20 & 336.7 & 8.14 & 1.97 & 0.276 & 0.884 & 0.0256 \\
\hline expt & - & - & $\begin{array}{l}1.953(3) \text { 46 } \\
\end{array}$ & \begin{tabular}{lll|l|}
$0.2859(3)$ & 67 & 68 & 69 \\
\end{tabular} & $\begin{array}{l}0.8781(44) \text { [4] } \\
\end{array}$ & $0.0256(4) \quad \overline{45}$ \\
\hline
\end{tabular}




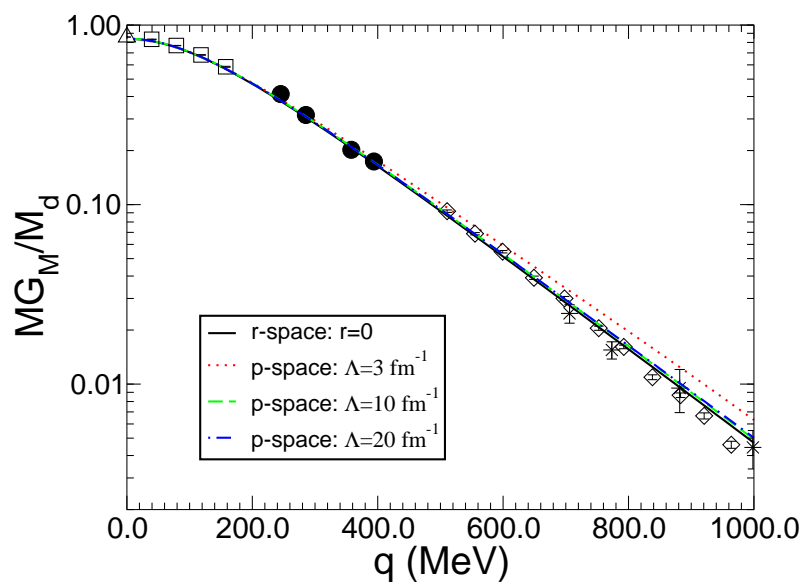

Figure 5. Magnetic form factor of deuterium $G_{M}$ obtained using different regularizations of the leading-order $\chi$ ET potential. Legend for theory curves as in Fig. 3. Experimental data from deuteron magnetic moment, open triangle 61; the parameterization of Ref. [3], open squares; and measurements of $B\left(Q^{2}\right)$ : solid circles [62, open diamonds 63], and stars 64].

These issues aside, we see that the LO result for all three form factors is in remarkably good agreement with the data. In fact, as $\Lambda \rightarrow \infty$, the agreement with the data improves consistently. Obviously, the unphysical, spurious bound states affect the structure of the wave functions only at distances which are not relevant for momentum transfers $|\mathbf{q}|$ below $1 \mathrm{GeV}$. The spurious bound states in the ${ }^{3} \mathrm{~S}_{1}-{ }^{3} \mathrm{D}_{1}$ channel have no impact on these observables in the domain of validity of $\chi \mathrm{ET}$. Consequently their appearance in the spectrum in no way signals a breakdown of this approach.

The agreement obtained between the data and the form factors evaluated with $\mathrm{LO}$ wave functions is surprising and also encouraging. One advantage of $\chi \mathrm{ET}$ over phenomenological models of deuterium is its ability to systematically improve its predictions. Therefore in the next section we examine what happens when certain sub-leading effects are included in the wave functions and also discuss the impact on the predictions of pieces of the current operator that are of higher chiral order.

\section{Impact of two-pion exchange and exchange currents on the form factors}

To begin our discussion of higher-order effects on the deuterium form factors we first discuss some of the higher-order contributions to the current operators. Both $J_{0}$ and $\mathbf{J}$ have a chiral expansion, and to produce the results displayed in the previous section we have ignored terms of order $\left(\frac{P}{\Lambda}\right)^{3}$. We have also dropped terms suppressed by $\left(\frac{P}{M}\right)^{2}$. In general corrections $\left(\frac{P}{\Lambda}\right)^{n}$ will be larger than corrections $\left(\frac{P}{M}\right)^{n}$, as emphasized in Weinberg's original power counting used, e.g., in [28,66. $\left(\frac{P}{\Lambda}\right)^{n}$ modifications to Eqs. (27)-(29) are associated with two-body operators because we have used phenomenological nucleon form factors. The strength of these terms is not fixed by any symmetry and must be determined by data (from either the single-nucleon or two-nucleon sector). While the first such effects in $G_{M}$ are of relative order $P^{3}$, the first occurrence of such a two-body operator in $G_{C}$ and $G_{Q}$ is not until relative order $P^{4}$. It is therefore higher-order than all the TPE effects in $V$ that we will consider here.

On the other hand, corrections to Eqs. (27) and (28) of nominal size $\left(\frac{P}{M}\right)^{n}$ occur in $J_{0}$ with $n=2$. These are corrections to the one-body part of $J_{0}$, and they can be obtained by demanding that matrix elements of the one-body current operator obey the correct transformation properties for Lorentz boosts to frames with velocities $v \ll c$. If we do this, we find a modification to the one-body part of $J_{0}$ that introduces an additional factor of $1-\frac{Q^{2}}{8 M^{2}}$ in Eq. (25). In addition, if we are to have low-energy Lorentz covariance for deuteron matrix elements of the current operator $\left(J_{0}, \mathbf{J}\right)$ the momentum transfer $|\mathbf{q}|$ that is used in evaluating the integrals of Eq. (27)-(29) must also be redefined. (This accounts for the boost of the deuteron wave function from the deuteron center-of-mass to the Breit frame 65. 26].) Both of these are small effects at the range of $Q^{2}$ values considered here. Specifically, the former does not affect the minimum of $\left|G_{C}\right|$ at all, while the latter shifts it to the right by $3 \%$, i.e. about $15 \mathrm{MeV}$ for the LO results presented above. A two-nucleon effect in $J_{0}$ that is of order $\frac{P^{3}}{\Lambda^{2} M}$ will be discussed explicitly below.

Because these $1 / M^{2}$ effects to the operator $J_{0}$ are generically small, in what follows we focus on the impact of improving the wave functions. In particular, we first want to apply the NNLO wave functions that were discussed in Sec. 2 and which incorporate corrections of size $\left(\frac{P}{\Lambda}\right)^{3}$. The procedure used to evaluate $G_{C}, G_{Q}$, and $G_{M}$, with these wave functions is the same as in Sec. 3. i.e. single-nucleon form factors of Belushkin, Hammer, and Meißner are incorporated in the calculation to account for the effects of single-nucleon structure as per Eqs. (27)-(29). 


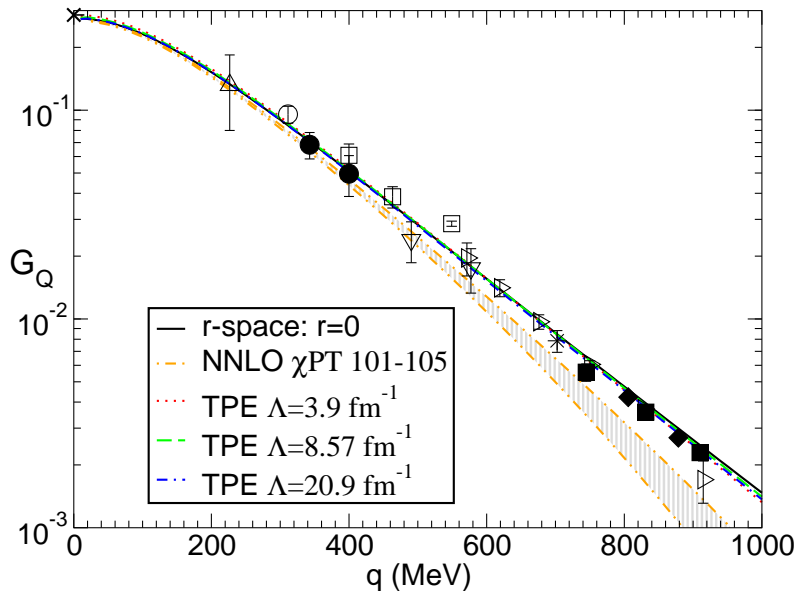

Figure 6. Quadrupole form factor of deuterium $G_{Q}$ obtained using using different regularizations of the TPE potential. Legend as in Fig. 3, with slight differences in cutoffs indicated.

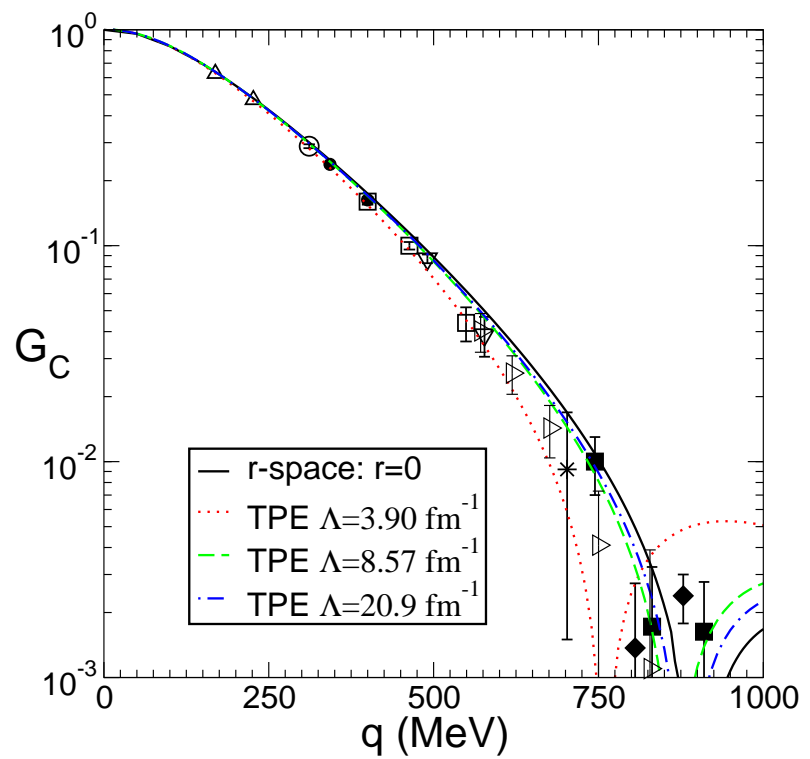

Figure 7. Charge form factor of deuterium $G_{C}$ obtained using using different regularizations of the TPE potential. Legend as in Fig. 3, with slight differences in cutoffs indicated.

The pattern of convergence to the $\Lambda \rightarrow \infty$ limit is very much the same as in the case of the OPE potential discussed in Sec. 3. Here, we first show results for $G_{C}$ and $G_{Q}$, in Figs. 6 and 7 respectively. $G_{Q}$ remains largely insensitive to $\Lambda$. At $|\mathbf{q}|=0$, the sensitivity is as large as that at any $|\mathbf{q}|$, and is approximately $3 \%$ over the range from $\Lambda=3.9$ fm ${ }^{1}$ to $\Lambda=14.1 \mathrm{fm}^{-1}$. Perhaps not coincidentally, this is roughly the size of the correction needed to move the value of $Q_{d}$ obtained with NNLO $\chi$ ET to the experimental result [27]. We find that $G_{C}$ is more sensitive to $\Lambda$. In particular, the cutoff dependence of $G_{C}$ around its zero is somewhat larger than naive expectations given the breakdown scale of $\chi \mathrm{PT}$, and so we stress that that such expectations might be misleading, especially in the vicinity of a zero like this. For both form factors, the limit as $\Lambda \rightarrow \infty$ of the results with the momentum-space cutoff is equal (within numerical uncertainties) to the result obtained via imposing a boundary condition in co-ordinate space at $r=0$.

In Fig. 6] we also show results for one of the modern $\chi \mathrm{PT} N N$ interactions of Ref. [66]. For this observable, we will show below that the uncertainties due to different sets of $\pi \mathrm{N}$ LECs, the nucleon form factor and different choices for $\eta$ are rather small. But Fig. 6 shows that results based on the NNLO $\chi$ ET wave functions of Ref. 66, which employ a regulator $\exp \left(-p^{6} / \Lambda^{6}\right)$ with $\Lambda$ in the range $500-700 \mathrm{MeV}$, do not overlap with the predictions from our wave functions at any $|\mathbf{q}|$.

Having demonstrated the equivalence of co-ordinate- and momentum-space formulations of TPE in the limit in which the regulator is removed, we now examine the impact of TPE on the form factors themselves by comparing results for the various sets of $c_{i}$ 's given in Table 1. We begin with results for $G_{C}$, which are presented in Fig. 8 , where 


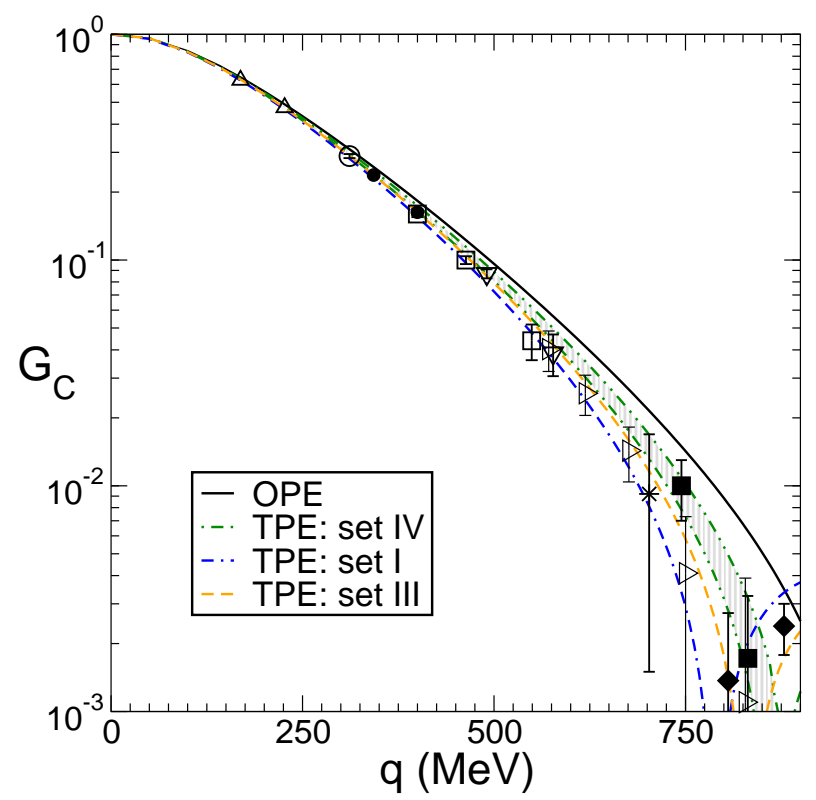

Figure 8. Comparison of charge form factor $G_{C}$ with (blue, green, and orange broken curves) and without (black solid curve) the NNLO corrections included in the $N N$ potential. The green double-dot-dashed, blue double-dash-dotted, and orange shortdashed curves use the different choices of the $c_{i}$ 's listed in Table 1 . The error bands in the theoretical calculation shown for the "Set IV" choice of $\pi$ N LECs incorporate the experimental uncertainties in both the input value of $\eta$ and the nucleon form factors of Ref. [49]. Data from the compilation of Ref. [51].

we also compare to data. Here we display the results for Sets I, III, and IV. (The results for Set II are very similar to those obtained with Set I.) The error band shown for the Set IV choice incorporates both uncertainties in the deuteron wave function (mainly those from varying the asymptotic $D / S$-ratio in this case), as well as the uncertainty bands in the single-nucleon form factors quoted in Ref. 49. Sensitivity of $G_{C}$ to these uncertainties is similar for the other two sets of $\pi \mathrm{N}$ LECs. We note that such uncertainties are smaller than the sensitivity to different choices for the $c_{i}$ 's.

The results clearly show that TPE corrections are small, as anticipated from the chiral expansion, and thatindependent of the $\pi \mathrm{N}$ LECs used to compute TPE effects - they shift the minimum of $\left|G_{C}\right|$ to the left. This yields a rather remarkable agreement between the $G_{C}$ obtained once TPE is included in the deuteron wave function and experimental data. We will see below that effects of similar size can be expected from exchange-current contributions to the operator $J_{0}$. Therefore, at this point, no further conclusions on the size of LECs in TPE can be drawn. However, the results clearly indicate that it will be interesting to study $G_{C}$ again taking such two-body effects in $J_{0}$ into account. The results of Fig. 8 also suggest that the minimum of $\left|G_{C}\right|$ could be a fruitful place to look for improvements in the EFT's description of data due to the inclusion of explicit $\Delta(1232)$ degrees of freedom. Such calculations are, however, beyond the scope of this study.

If the NNLO potential is used to compute deuteron wave functions the deuteron's asymptotic D-to-S ratio, $\eta$, is a free parameter of the calculation. In Fig. 9 we show how the position of $\left|G_{C}\right|$ 's minimum depends on $\eta$. Note that in order to make such a plot we need to specify the long-range potential, i.e. the set of $c_{i}$ 's we used for TPE. Also shown in Fig. 9] is the regulator-independent result obtained with the OPE potential, where $\eta$ is not a free parameter, but is determined by the dynamics. This figure demonstrates that the position of the $\left|G_{C}\right|$ minimum is only weakly dependent on $\eta$. In particular, had we used $\eta_{\mathrm{OPE}}(\gamma) \approx 0.026333$ as our input instead of the central experimental value $\eta_{\text {expt }}=0.0256$ the minimum would only have shifted about $4 \%$ to the right. But the overall shift in the minimum from the OPE result to the TPE result with Set IV $c_{i}$ 's is $12 \%$ (the shifts for Set I-III $c_{i}$ 's are even larger). So roughly two-thirds of the shift in the $\left|G_{C}\right|$ minimum arises from the inclusion of TPE and corresponding changes of the wave function at distances $r \sim 1 / m_{\pi}$. When regulator-independent wave functions are employed two-pion exchange has a significant impact on the $|\mathbf{q}|$ at which the zero of $G_{C}$ occurs, regardless of which $c_{i}$ 's are used in its evaluation.

Turning our attention now to Fig. 10, we see that the OPE and TPE results for $G_{Q}$ are very close together. Above $|\mathbf{q}|=350 \mathrm{MeV}$ the effect of TPE is to shift $G_{Q}$ downwards, irrespective of the set of $c_{i}$ 's chosen. However, the error bands on the OPE and TPE calculations are always overlapping (recall that though we only show the error band for the TPE: Set IV calculation, all three TPE calculations have bands of similar size due to uncertainties in their input). The only place where an unambiguous difference between the different $N N$ potentials can be seen is in the quadrupole moment. But here it is difficult to draw any definitive conclusion, since all calculations have central values that under-predict the experimental result $Q_{d}=0.2859(3) \mathrm{fm}^{2}[67,68$. This discrepancy is, however, consistent in all 


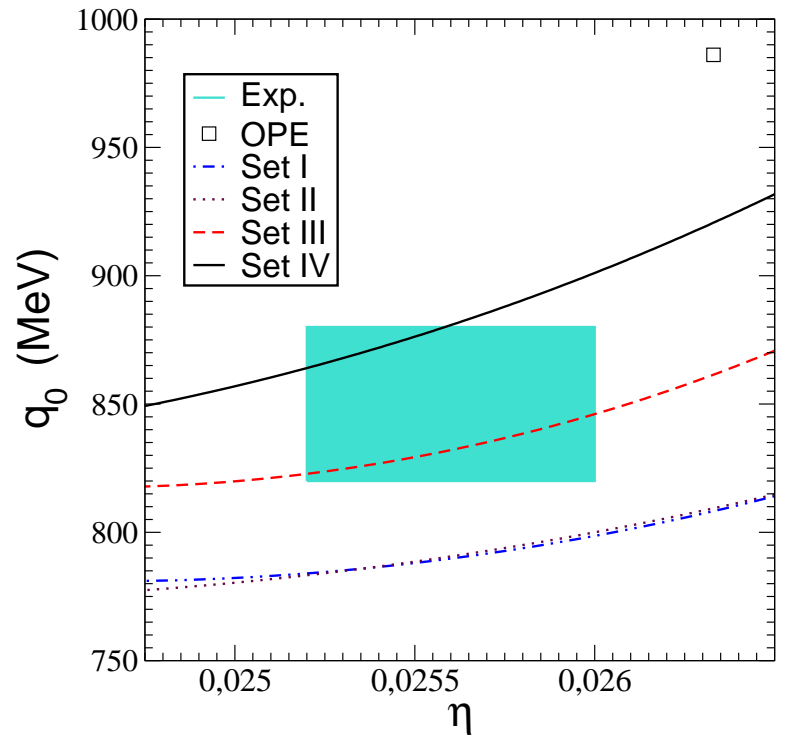

Figure 9. The position of the zero in the charge form factor, $q_{0}$, as a function of the asymptotic D-to-S ratio $\eta$ for the four different sets of $\pi \mathrm{N}$ LECs considered here. The $(1 \sigma)$ experimental constraints on $\eta$ and $q_{0}$ are indicated by the shaded region. The result found for $\eta$ and $q_{0}$ from the LO $\chi \mathrm{ET}$ potential with $\Lambda \rightarrow \infty$ is given by the square.

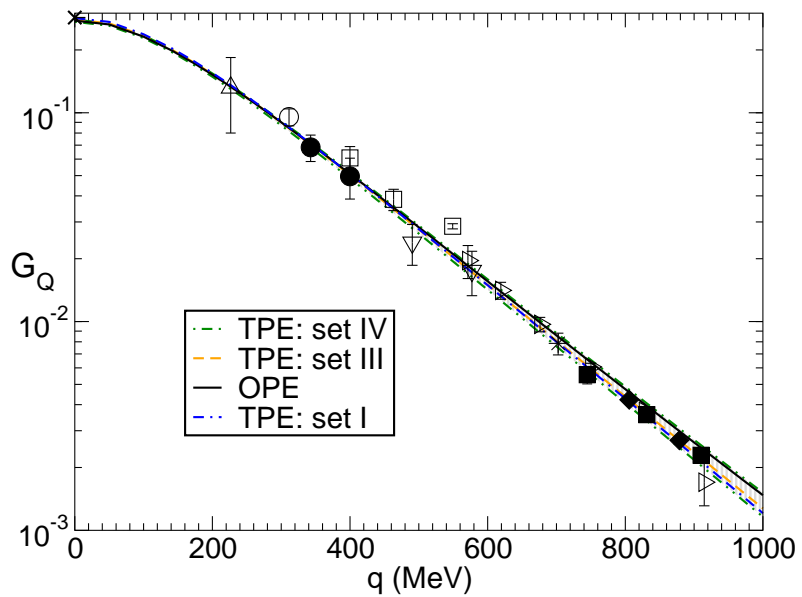

Figure 10. Comparison of quadrupole form factor $G_{Q}$ with (blue, orange, and green broken curves) and without (black solid curve) the NNLO corrections included in the $N N$ potential. The green double-dot-dashed, blue double-dash-dotted, and orange short-dashed curves use the different choices of the $c_{i}$ 's listed in Table 1 The error bands in the theoretical calculation shown for the "Set IV" choice of $\pi \mathrm{N}$ LECs incorporate the experimental uncertainties in both the input value of $\eta$ and the nucleon form factors of Ref. [49. Data from the compilation of Ref. [51].

cases with the expected short-distance contribution to $G_{Q}$ which occurs at $O\left(e P^{5}\right)$ in the chiral expansion for $J_{0}$. All of this suggests that $G_{Q}$ is not a good place to attempt to test the TPE contribution to the deuteron wave function.

In contrast, $G_{M}$ is quite sensitive to the choice of dynamics for the long-range part of the $N N$ potential, as is seen in Fig. 11. The uncertainties from the input value of $\eta$ and the single-nucleon form factors are sizeable, but even allowing for these uncertainties there are differences between OPE and TPE wave functions at the 1-2 $\sigma$ level. And at least at $|\mathbf{q}|>0.5 \mathrm{GeV}$, there is also significant sensitivity to the choice of $\pi \mathrm{N}$ LECs that is employed in the sub-leading TPE. Unfortunately the effect of TPE here is to worsen the excellent agreement with data that is achieved with OPE. (Although we note that Set IV is still marginally consistent with data if we allow for all input uncertainties.) However, it should be remembered that the theoretical uncertainty due to higher-order corrections in $G_{M}$ is not depicted here. Such effects in the current $\mathbf{J}$ are suppressed by $P^{3} / \Lambda^{3}$ relative to leading, the same relative size as TPE, and so should be included if a consistent calculation up to that order of $G_{M}$ in the chiral expansion is desired. Until such a calculation is completed only tentative conclusions about the impact of the $O\left(P^{3}\right)$ pieces of the $N N$ potential on $G_{M}$ can be drawn. 


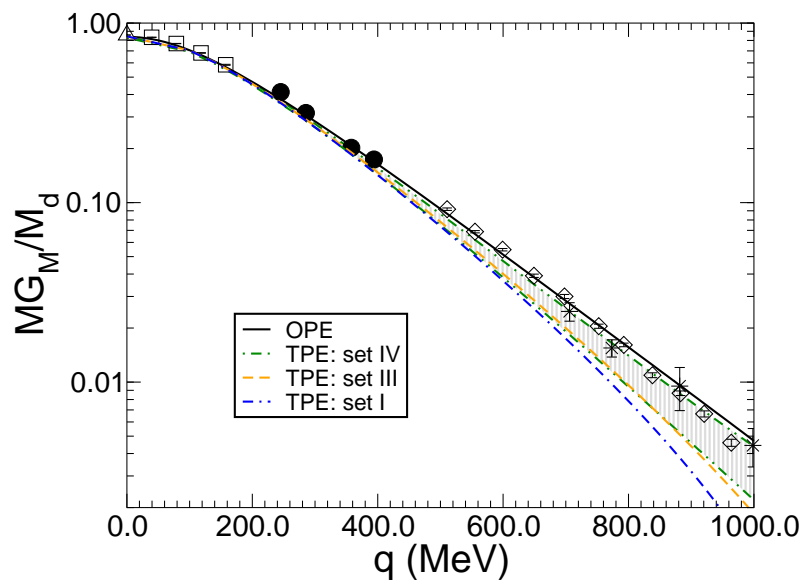

Figure 11. Comparison of magnetic form factor $G_{M}$ with (blue, orange, and green broken curves) and without (black solid curves) the NNLO corrections included in the $N N$ potential. The green double-dot-dashed, blue double-dash-dotted, and orange short-dashed curves use the different choices of the $c_{i}$ 's listed in Table 1 The error bands in the theoretical calculation shown for the "Set IV" choice of $\pi \mathrm{N}$ LECs incorporate the experimental uncertainties in both the input value of $\eta$ and the nucleon form factors of Ref. [9]. Data as in Fig. [5]

Similar caution is advisable in the interpretation of our results for $G_{C}$ and $G_{Q}$. In this case there is a two-body piece of $J_{0}$ at relative order $P^{3}$ whose coefficient is fixed by the low-energy consequences of Lorentz covariance. This operator was first derived in Ref. [70, and was re-derived in $\chi \mathrm{ET}$ in Ref. [26]. The two-body matrix element of this piece of $J_{0}$ can be computed reliably with the momentum-space wave functions of Section 2, It converges to a definite result as $\Lambda \rightarrow \infty$ and shifts the minimum of $\left|G_{C}\right|$ to the left by about $100 \mathrm{MeV}$, worsening the agreement with data seen in Fig. 8, However, as emphasized by Friar [71] and Adam and Arenhövel 72, this correction to $J_{0}$ is associated with $P^{2} / M^{2}$ corrections to the OPE potential used in generating the wave functions. None of those $P^{2} / M^{2}$ pieces of $V$ were included in our calculations. Therefore a full evaluation of the relevant $1 / M$-suppressed effects remains to be performed with these wave functions. Such an evaluation is, however, beyond the scope of this paper.

\section{Conclusion}

Electron-deuteron scattering provides a window onto $N N$ dynamics that gives complementary information to that obtained in $N N$ scattering. It allows us to work at fixed total energy of the $N N$ system and examine the electromagnetic response of the system as a function of the momentum-transfer squared, $q^{2}$ (which is equal to $-|\mathbf{q}|^{2}$ in the Breit frame). In $\chi \mathrm{ET}$ the predictions for the three electromagnetic form factors $G_{C}, G_{Q}$, and $G_{M}$ that determine all elastic electrondeuteron scattering observables in the one-photon-exchange approximation are given solely by single-nucleon operators up to corrections of relative order $P^{3}$. Consequently the venerable formulae (27)-(29) for these form factors as Bessel transforms of probability densities are valid to this level of accuracy.

Examination of these formulae indicates that the charge form factor is sensitive to distances up to a value of $r$ given by

$$
r \approx \pi / \frac{|\mathbf{q}|}{2}
$$

where the factor of $\pi$ upstairs arises from the first zero of $j_{0}(x)$ and the factor of $1 / 2$ in the momentum transfer occurs because only half of $\mathbf{q}$ is transmitted to the relative degree of freedom. Thus as we move through the momentum range $0 \leq|\mathbf{q}| \leq 1 \mathrm{GeV} G_{C}$ changes from an average of the probability density over all $r$ to one where the important dynamics is that taking place at $r \approx 1.2 \mathrm{fm}$. This radius is well inside that at which OPE is active, and indeed is small enough that some TPE effects are probed. Once $|\mathbf{q}| \approx 1 \mathrm{GeV}$ we expect that two-body operators which scale as $\left(\frac{|\mathbf{q}|}{\Lambda_{\chi \mathrm{SB}}}\right)^{n}$ with $n \geq 3$ will no longer be suppressed, and so the dominance of the single-nucleon contributions to the deuteron four-current is no longer guaranteed there. But the momentum range $|\mathbf{q}| \leq 1 \mathrm{GeV}$ already provides a wide kinematic domain over which we can test $\chi$ ET predictions for the deuteron wave functions (or equivalently - up to $P^{3}$ corrections - form factors), and examine the impact of regularization on these predictions.

Several calculations of these form factors that use $\chi$ ET already exist in the literature $24,25,26,27$. But here we focused particularly on the possibility to obtain regulator-independent predictions for $G_{C}, G_{Q}$, and $G_{M}$ in the regime $|\mathbf{q}| \leq 1 \mathrm{GeV}$. We found that the results for all three form factors become stable as the momentum-space cutoff on the $\chi \mathrm{ET}$ calculation, $\Lambda$, is taken to infinity. The resulting predictions are therefore free of artifacts due to the particular 
function that is chosen as a short-distance regulator. They can also be obtained by employing a co-ordinate-space wave function where the Schrödinger equation is solved for all $r>0$ using $N N$ potentials derived from chiral perturbation theory. We found that the momentum-space cutoff must be taken to be more than $2 \mathrm{GeV}$ before stability is obtained in some cases (e.g. $G_{C}$ near its zero, $G_{M}$ at $|\mathbf{q}| \approx 0.45 \mathrm{GeV}$ ). We therefore caution that invoking naive-dimensionalanalysis estimates to set the size of cutoffs that should be employed in $\chi \mathrm{ET}$ can lead to the presence of significant cutoff artifacts in the results for observables.

We were able to perform these calculations for both the LO $N N$ potential (which consists solely of one-pion exchange at long range) and for the NNLO $N N$ potential. Because both of these potentials are singular and attractive as $r \rightarrow 0$ they can generate a shallow (deuteron) bound state. But their singular nature means that as $\Lambda \rightarrow \infty$ they also generate spurious bound states at energies beyond the range of applicability of the theory. However, these spurious bound states have no impact on the deuteron form factors, and we found no indication that their appearance signals a breakdown of the $\chi \mathrm{ET}$ approach.

We were not able to perform a calculation with the NLO $\chi \mathrm{PT} N N$ potential, since the potential at this order is singular and repulsive. In consequence it is impossible to take the $\Lambda \rightarrow \infty$ limit with this $V$ inserted in the Schrödinger equation and still have a spectrum with a shallow bound state. An order-by-order assessment of the convergence of $\chi$ ET must therefore await the development of a power counting which does not have this deficiency. In this context we note that the version of $\chi \mathrm{PT}$ in which the $\Delta(1232)$ is included as an explicit degree of freedom leads to a potential at NLO in the (modified) chiral expansion that is singular and attractive. Indeed, in the ${ }^{3} \mathrm{~S}_{1}-{ }^{3} \mathrm{D}_{1}$ channel the NLO potential in the theory with explicit Deltas is very similar to the NNLO potential we have used here. Therefore we believe that a study employing the NLO $V$ from $\chi \mathrm{PT}$ with explicit Deltas - while definitely called for-will likely reach similar conclusions to those found here using the NNLO $V$ from standard (Delta-less) $\chi \mathrm{PT}$.

The calculations to LO in $\chi \mathrm{ET}$ gave predictions for deuteron electromagnetic structure which are remarkably close to experiment for $|\mathbf{q}| \leq 0.6 \mathrm{GeV}$ once the $\Lambda \rightarrow \infty$ limit was taken. The only significant disagreement between the LO prediction and the data in the compilation of Ref. [51] occurred in the vicinity of the zero of $G_{C}$. But even there the discrepancy between theory and data was within the expected $\left(\frac{Q^{2}}{4 \Lambda_{\chi \mathrm{SB}}^{2}}\right)$, which is about $25 \%$ at $1 \mathrm{GeV}$.

The corrections to the LO result once the NNLO potential was employed to compute deuteron structure were also consistent with this expectation, proving to be small, especially in the case of $G_{Q}$, which is already well described by the LO calculation. Our results also show that the discrepancy between the LO $\chi \mathrm{ET}$ calculation and experimental data for $G_{C}$ in the vicinity of its zero may be eliminated when such a higher-order calculation is performed. In particular, we showed that the position of the minimum in $G_{C}$ is sensitive to details of the two-pion-exchange interaction that is present in the NNLO $\chi \mathrm{PT}$ potential. This will be an interesting point to focus on in future investigations of twopion exchange. In contrast, the good description of $G_{M}$ in LO appears to be accidental. Various small but significant higher-order effects apparently cancel for this observable, but this needs to be checked in a complete higher-order calculation.

We also assessed the impact of the $O\left(P^{3}\right)$ corrections to $J_{0}$ on our results for $G_{C}$ and found that they converge to a definite limit as $\Lambda \rightarrow \infty$. Although we did not include any $\chi \mathrm{PT}$ corrections to the leading-order $J_{\mu}$ in the results we presented, our estimates of the $O\left(P^{3}\right)$ effects in $J_{0}$ show that the exchange-current corrections have roughly the same impact on $G_{C}$ in the vicinity of its zero as do the $O\left(P^{3}\right)$ contributions to $V$. This suggests that the power counting for the potential and the currents is working well in this domain.

All of this makes it very interesting to complete consistent higher-order calculations of deuteron form factors using these regulator-independent wave functions. To do this the $O\left(P^{3}\right)$ pieces of $J_{\mu}$ will have to be computed fully and in a manner that is consistent with the treatment of $1 / M^{2}$ corrections in $V$. Only when such a computation is performed will we know if the improvement found here with the NNLO $\chi \mathrm{ET}$ deuteron wave functions represents genuinely good convergence of the chiral expansion for these observables or not. The success we observed here may be merely a fortunate result of examining only the corrections to $V$. And an assessment of the impact that $O\left(P^{4}\right)$ (and higher) pieces of $J_{0}$ will also be necessary if a definitive conclusion as regards the impact of TPE contributions on the position of $G_{C}$ 's zero is to be reached. Irrespective of these issues, though, it is clear that the deuteron charge form factor is sensitive to details of the chiral dynamics that is at work in deuterium.

We are grateful to the organizers of the Trento workshop on "QCD and Nuclear Forces: Never the Twain Shall Meet?" (June 2005) for providing a stimulating environment which enabled us to come together and initiate this research. We thank Maxim Belushkin for providing the nucleon form factor data files, and Evgeny Epelbaum, Ulf Meißner and Matthias Schindler for comments on the manuscript.

The work of DP was supported under US Department of Energy grant DE-FG02-93ER40756. The work of ERA is supported in part by funds provided by the Spanish DGI and FEDER funds with grant no. FIS2005-00810, Junta de Andalucía grants no. FQM225-05, EU Integrated Infrastructure Initiative Hadron Physics Project contract no. RII3-CT-2004-506078. MPV has been funded by the Deutsche Forschungsgemeinschaft (SFB/TR 16), Helmholtz Association (contract number VH-NG-222). Part of the numerical calculations have been performed on JUMP and JUBL of the JSC in Jülich, Germany. 


\section{References}

1. R. Gilman and F. Gross, J. Phys. G 28, R37 (2002).

2. M. Garcon and J. W. Van Orden, Adv. Nucl. Phys, 26, 293 (2001).

3. I. Sick, Prog. Part. Nucl. Phys. 47, 245 (2001).

4. V. Bernard and U.-G. Meißner, arXiv:hep-ph/0611231

5. S. Scherer and M. R. Schindler, arXiv:hep-ph/0505265

6. S. Weinberg, Phys. Lett. B 251, 288 (1990).

7. S. Weinberg, Nucl. Phys. B 363, 3 (1991).

8. S. Weinberg, Phys. Lett. B 295, 114 (1992).

9. S. R. Beane, P. F. Bedaque, W. C. Haxton, D. R. Phillips and M. J. Savage, arXiv:nucl-th/0008064

10. P. F. Bedaque and U. van Kolck, Ann. Rev. Nucl. Part. Sci. 52, 339 (2002).

11. E. Epelbaum, Prog. Part. Nucl. Phys. 57, 654 (2006).

12. D. R. Phillips, arXiv:0710.3597 [nucl-th].

13. M. Pavón Valderrama and E. Ruiz Arriola, Phys. Rev. C 74, 064004 (2006) [Erratum-ibid. C 75, 059905 (2007)].

14. A. Nogga, R. G. E. Timmermans and U. van Kolck, Phys. Rev. C 72, 054006 (2005).

15. E. Epelbaum and U.-G. Meißner, arXiv:nucl-th/0609037

16. M. C. Birse, Phys. Rev. C 76, 034002 (2007).

17. S. R. Beane, P. F. Bedaque, M. J. Savage and U. van Kolck, Nucl. Phys. A 700, 377 (2002).

18. M. Pavón Valderrama and E. Ruiz Arriola, Phys. Rev. C 72, 054002 (2005).

19. C. J. Yang, C. Elster and D. R. Phillips, arXiv:0706.1242 [nucl-th].

20. K. M. Case, Phys. Rev. 80, 797 (1950).

21. M. Pavón Valderrama and E. Ruiz Arriola, Phys. Rev. C 74, 054001 (2006).

22. M. Rho, Phys. Rev. Lett. 66, 1275 (1991).

23. T. S. Park, D. P. Min and M. Rho, Nucl. Phys. A 596, 515 (1996).

24. D. R. Phillips and T. D. Cohen, Nucl. Phys. A 668, 45 (2000).

25. M. Walzl and U.-G. Meißner, Phys. Lett. B 513, 37 (2001).

26. D. R. Phillips, Phys. Lett. B 567, 12 (2003).

27. D. R. Phillips, J. Phys. G 34, 365 (2007).

28. C. Ordóñez, L. Ray and U. van Kolck, Phys. Rev. C 53, 2086 (1996).

29. N. Kaiser, R. Brockmann and W. Weise, Nucl. Phys. A 625, 758 (1997).

30. E. Epelbaum, W. Glöckle and U.-G. Meißner, Nucl. Phys. A 671, 295 (2000).

31. N. Kaiser, S. Gerstendorfer and W. Weise, Nucl. Phys. A 637 (1998) 395.

32. H. Krebs, E. Epelbaum and U.-G. Meißner, Eur. Phys. J. A 32, 127 (2007).

33. A. Nogga and C. Hanhart, Phys. Lett. B 634, 210 (2006).

34. S. R. Beane, P. F. Bedaque, M. J. Savage and U. van Kolck, Nucl. Phys. A 700, 377 (2002).

35. D. W. L. Sprung, W. van Dijk, E. Wang, D. C. Zheng, P. Sarriguren and J. Martorell, Phys. Rev. C 49, 2942 (1994).

36. L. Platter and D. R. Phillips, Phys. Lett. B 641, 164 (2006).

37. U. van Kolck, Nucl. Phys. A 645, 273 (1999).

38. D. R. Entem, E. Ruiz Arriola, M. Pavón Valderrama, R. Machleidt, arXiv:0709.2770 nucl-th].

39. J. L. Friar, Phys. Rev. C 60, 034002 (1999).

40. M. C. M. Rentmeester, R. G. E. Timmermans, J. L. Friar and J. J. de Swart, Phys. Rev. Lett. 82, 4992 (1999).

41. V. Bernard, N. Kaiser and U.-G. Meißner, Nucl. Phys. A 615, 483 (1997).

42. P. Büttiker and U.-G. Meißner, Nucl. Phys. A 668, 97 (2000).

43. E. Epelbaum, W. Glöckle and U.-G. Meißner, Eur. Phys. J. A 19, 401 (2004).

44. D. R. Entem and R. Machleidt, Phys. Rev. C 68, 041001 (2003).

45. N. L. Rodning and L. D. Knutson, Phys. Rev. C 41, 898 (1990).

46. S. Klarsfeld, J. Matorell, J.A. Oteo, M. Nishimura, and D.W.L. Sprung, Nucl. Phys. A456, 373 (1986).

47. I. Borbély, W. Grüebler, V. König, P.A. Schmelzbach, and A.M. Mukhamedzhanov, Phys. Lett. 160B, 17 (1985).

48. M. Pavón Valderrama and E. Ruiz Arriola, arXiv:nucl-th/0605078.

49. M. A. Belushkin, H. W. Hammer and U.-G. Meißner, Phys. Rev. C 75, 035202 (2007).

50. R. Schiavilla and I. Sick, Phys. Rev. C 64, 041002 (2001).

51. D. Abbott et al., Eur. Phys. J. A7, 421 (2000).

52. D. M. Nikolenko et al., Phys. Rev. Lett. 90, 072501 (2003).

53. V. F. Dmitriev et al., Phys. Lett. B. 157, 143 (1985).

54. M. Ferro-Luzzi et al., Phys. Rev. Lett. 77, 2630 (1996).

55. M. E. Schulze et al., Phys. Rev. Lett. 52, 597 (1984).

56. M. Bouwhuis et al., Phys. Rev. Lett. 82, 3755 (1999).

57. R. Gilman et al., Phys. Rev. Lett. 65, 1733 (1990).

58. B. Boden et al. Z. Phys. C49, 175 (1991).

59. M. Garcon et al., Phys. Rev. C49, 2516 (1994).

60. D. Abbott et al., Phys. Rev. Lett. 84, 5053 (2000).

61. I. Lindgren in Alpha, Beta, and Gamma-Ray Spectroscopy, Vol. 2, ed. K. Siegbahn (North Holland, Amsterdam, 1965). 
62. G. G. Simon and C. Schmitt and V. H. Walther, Nucl. Phys. A364, 285 (1981).

63. S. Auffret et al., Phys. Rev. Lett. 54, 649 (1985).

64. R. Cramer et al., Z. Phys. C29, 513 (1985).

65. J. Adam and H. Arenhövel, Nucl. Phys. A614, 289 (1997).

66. E. Epelbaum, W. Glöckle, and U.-G. Meißner, Nucl. Phys. A 747, 362 (2005);

67. R. F. Code and N. F. Ramsey, Phys. Rev. A 4, 1945 (1971).

68. D. M. Bishop and L. M. Cheung, Phys. Rev. C 20, 381 (1979).

69. T. E. O. Ericson and M. Rosa-Clot, Nucl. Phys. A 405, 497 (1983).

70. D. O. Riska, Prog. Part. Nucl. Phys. 11, 199 (1984).

71. J. L. Friar, Phys. Rev. C 22, 796 (1980).

72. J. Adam, H. Goller, and H. Arenhövel, Phys. Rev. C 48, 470 (1993). 